\title{
Representational momentum in scenes: Learning spatial layout
}

\author{
MARGARET P. MUNGER, MATTHEW C. DELLINGER, TRAVIS G. LLOYD, \\ KATHERINE JOHNSON-REID, NICOLE J. TONELLI, KATHARINE WOLF, and JASON M. SCOTT \\ Davidson College, Davidson, North Carolina
}

\begin{abstract}
In four experiments, we examined whether watching a scene from the perspective of a camera rotating across it allowed participants to recognize or identify the scene's spatial layout. Completing a representational momentum (RM) task, participants viewed a smoothly animated display and then indicated whether test probes were in the same position as they were in the final view of the animation. We found RM anticipations for the camera's movement across the scene, with larger distortions resulting from camera rotations that brought objects into the viewing frame compared with camera rotations that took objects out of the viewing frame. However, the RM task alone did not lead to successful recognition of the scene's map or identification of spatial relations between objects. Watching a scene from a rotating camera's perspective and making position judgments is not sufficient for learning spatial layout.
\end{abstract}

When asked to remember the final position of a moving object, participants accept test probes that are placed farther along the object's trajectory, as if participants' representation of the movement is anticipating the immediate future. This positive distortion has been labeled representational momentum (RM) (Freyd \& Finke, 1984) and has been used to explore various aspects of our representations of object motion (see Hubbard, 1995, 2005, for a review, and Thornton and Hubbard, 2002, for additional recent work). This anticipation of object movement has also been observed with scenes (DeLucia \& Maldia, 2006; Munger, Owens, \& Conway, 2005; Thornton \& Hayes, 2004). When participants are shown a scene moving across their field of vision, either smoothly animated (Thornton \& Hayes, 2004) or as a series of still images (DeLucia \& Maldia, 2006; Munger et al., 2005), they accept probes placed farther along the depicted trajectory of the objects in the scene, suggesting that their representation of the event includes the motion. The purpose of the present work is to examine whether participants also include the layout of the scene in their representation of the event.

Moving through a scene can certainly contribute to learning its layout (Christou \& Bülthoff, 1999; Gaunet,

We thank Tim Hubbard, Jay Rueckl, and two anonymous reviewers for helpful comments and the students of Psychology 301, Spring 2003, for assistance with data collection. Experiment 2 was presented at the Psychonomic Society meeting, November 2003, and Experiments 1, 3, and 4 were presented at the Eastern Psychological Association meeting, April, 2004. Correspondence concerning this article should be addressed to M. P. Munger, Department of Psychology, Davidson College, Box 7001, Davidson, NC 28035-7001 (e-mail: mamunger@davidson.edu).

Note-This article was accepted by the previous editorial team, when Colin M. MacLeod was Editor.
Vidal, Kemeny, \& Berthoz, 2001; Wilson \& Péruch, 2002). Because controlling views in real-world scenes is difficult, researchers typically develop virtual environments. Participants are allowed to explore the virtual environment and then are tested on their memory for spatial layout using orienting or map-drawing tasks. Both active exploration and passive viewing of a virtual environment allow participants to develop an understanding of the scene's layout (Christou \& Bülthoff, 1999; Gaunet et al. 2001; Wilson \& Péruch, 2002). The RM task is like the passive viewing condition, since participants do not control the motion (for an exception, see Jordan \& Knoblich, 2004).

Wilson and Péruch (2002) examined the role of attention in learning spatial layout using a virtual environment with several walls and objects. Half of the participants were instructed to remember the locations of the interior rooms, whereas the other half were instructed to remember the particular objects. The instructions given mattered: Participants instructed to attend to the spatial layout drew significantly better maps, and those instructed to attend to the particular objects had higher object recognition scores. With an RM task, participants are asked to attend to the position of a moving object, and the positive distortion they attribute to the object suggests that its motion is part of their representation (Freyd, 1993; Freyd \& Finke, 1984; Freyd \& Jones, 1994; Hubbard, 1995; Munger, Solberg, \& Horrocks, 1999; Thornton \& Hayes, 2004). The present study investigated whether the RM task directs attention to the spatial layout of the surrounding scene.

Most RM studies have involved single objects, precluding any possibility of relations between objects. However, RM distortions are influenced by the presence of additional objects in the display (Actis-Grosso \& Stucchi, 2003; Hubbard \& Bharucha, 1988; Hubbard 
\& Motes, 2005; Hubbard \& Ruppel, 1999; Munger \& Owens, 2004). When a small moving target is placed inside a frame, smaller and even backwards distortions of its movement occur when it vanishes near the frame's border (Actis-Grosso \& Stucchi, 2003; Hubbard \& Bharucha, 1988; Hubbard \& Motes, 2005). It has been suggested that this distortion reflects participants' expectations either that the target bounced (Hubbard \& Bharucha, 1988) or that it would not continue past the frame (Hubbard \& Motes, 2005). In contrast, when a much larger object is placed in the center of the display, distortions for a small moving target are displaced toward this landmark, regardless of the target's direction (Hubbard \& Ruppel, 1999). Finally, the sudden appearance of a second object as the moving target reaches its final position can lead to larger RM distortions (Munger \& Owens, 2004). Taken together, these rather different findings suggest that RM is sensitive to the placement of objects in simple scenes.

There is some more direct evidence that the RM task in a scene affects a participant's representation of the scene. Munger et al. (2005) looked at RM and a distortion for scenes called boundary extension (BE). BE occurs when participants are shown a close-up of a scene and asked to remember the entire scene. Despite careful instructions and even warnings, participants claim to remember more of the background than was actually presented (see, e.g., Intraub, 2002). BE has been shown to occur uniquely for scene boundaries and not for object boundaries (Gottesman \& Intraub, 2004) and appears to be a general aspect of spatial cognition (Intraub, 2004). Munger et al. examined $\mathrm{BE}$ ratings after participants had viewed a series of pictures depicting translations into scenes - that is, progressively closer close-ups - and found striking individual differences.

Participants who showed BE for single views during a baseline condition showed significantly less BE following a series of pictures depicting an approach - as if they were including the approaching motion in their representation of the scene, in keeping with RM. However, participants who had no BE for the single views had the opposite response following the approach: Their picture ratings revealed $\mathrm{BE}$. For both groups, the implied movement into the scene altered their memory for the final view, but in opposite ways. Some individuals quite readily extended the boundaries of a limited view, and when motion was included they appeared to add that information to their representation. However, Munger et al. (2005) also found that some individuals seemed to include information beyond the limited view only following an approach sequence, when they really had seen more of the surrounding scene. Implied movement into a scene may lead to a representation that includes the spatial layout, or surrounding scene, but this is not always the case. To the extent that BE reflects a representation of the surrounding scene, the addition of movement only sometimes enhances this representation (Munger et al., 2005).

To further examine the extent to which movement through a scene supports an understanding of the scene's layout, we wanted a more direct measure of spatial layout.
Spatial layout can be measured in a variety of ways, including the indication of particular locations within the scene, scene recognition, and various mapping tasks (Christou \& Bülthoff, 1999; Gaunet et al., 2001; Janzen, Schade, Katz, \& Herrmann, 2001; Wilson \& Péruch, 2002). In the present study, we used two measures that featured different visual information (see Figure 1). In the observer map task, participants indicated which of two different maps of the scene was correct. This perspective has been called the observer view (Janzen et al., 2001), reflecting the fact that the viewer is outside the scene-observing but not participating (see Figure 1A). Our other measure of spatial layout involved showing participants views of the objects identical to views seen during the RM task and asking participants to identify the object's neighbors (see Figure 1B). This perspective has been called the field view (Janzen et al., 2001), reflecting the fact that the viewer is inside the scene, or on the field. Janzen et al. examined the role of visual perspective (observer vs. field) in wayfinding and found that participants adopted different strategies depending upon how the scene was initially presented to them. Participants explored a series of corridors that included unusual oblique corridors along with traditional junctions with right angles. Participants first introduced to the observer perspective took advantage of the larger view, correctly including the obliquely angled corridors in

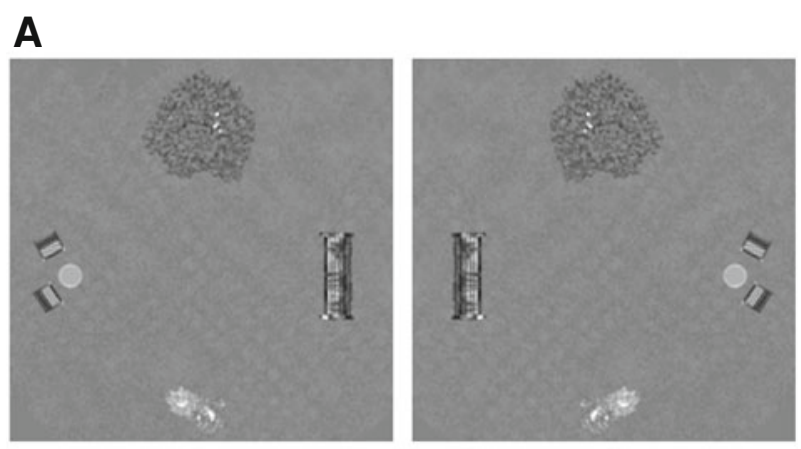

B

Which map is correct?

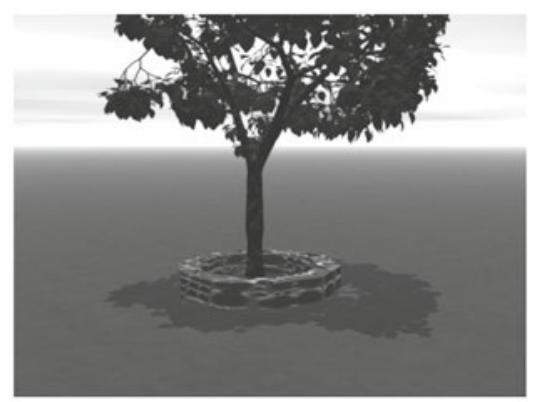

Here's the tree, what is to your right?

Figure 1. (A) The observer maps used for the recognition task in Experiments 2 and 4 (the map on the left is correct). (B) An example of the field view used: to test participants' memory for the map just memorized (Experiment 2), for a surprise test of layout (Experiment 3), and for the layout trials (Experiment 4). 
both mapping and wayfinding tasks. Participants using the field view, however, focused primarily on right angles.

Wilson and Péruch (2002) found that attention, not interactivity with the display, impacted learning of a scene's layout. In their study, participants instructed to attend to spatial layout produced better maps, whereas those attending to particular objects had higher object recognition scores. The RM task directs participants' attention to the final location of a moving object. Our main interest was in determining what aspects of the surrounding layout of the scene participants knew following this repeated judgment of position. In the experiments reported here, a digitally represented park scene (see Figure 2A), populated with some stationary objects and rendered with ground and sky (see Figure 2B), was presented from the perspective of a camera rotating at the center of the scene. Experiment 1 confirmed that this kind of rotational camera movement leads to RM distortions, since previous work has looked only at translations (DeLucia \& Maldia, 2006; Munger et al., 2005; Thornton \& Hayes, 2004). Experiment 2 used an observer's distanced perspective to assess participants' knowledge of the spatial layout, whereas Experiment 3 used the more intimate field view. Finally, Experiment 4 included a new task within the RM block designed to focus attention on the spatial layout of the depicted scene.

\section{EXPERIMENT 1}

Thornton and Hayes (2004) established that participants can anticipate the action in a visually complex scene both when a camera captures the action of moving objects and when the camera itself is moving into the scene (see also DeLucia \& Maldia, 2006; Munger et al., 2005). Our first task was to demonstrate that RM would occur when a camera rotated across a scene. A park scene with four distinct object groupings located around its perimeter was developed, with the camera placed in the center (see Figure 2). Movies resulting from the camera's being rotated, in a manner similar to turning one's head to look left or right, were used for the inducing displays (see Figure 3). Our first purpose was to see if camera rotations would also lead to RM anticipation in scenes. Each of our movies ended with an object group centered in the view; a new scene was then displayed, and participants indicated whether the presented probe was identical to the final position of the object group in the movie.

\section{Method}

Participants. Twenty undergraduates ( 7 men and 13 women) enrolled in introductory psychology at Davidson College participated for partial course credit.

Apparatus. The stimuli were displayed and data were collected by a Power Macintosh G4 Cube computer. Subjects sat at a comfortable viewing distance (approximately $50 \mathrm{~cm}$ ) from a 17-in. Apple Studio Display monitor, set at a $99-\mathrm{Hz}$ refresh rate.

Stimuli. A park scene was developed using Bryce 3-D that featured four distinct object groups around a perimeter: a red-leafed tree; a red table and blue chairs; two large planters, one with green plants and the other with pink flowers; and a wooden bench (see Figure 2). When the camera was centered on a single object group, none of the other three object groups was visible. In other words, when the bench was centered in view, neither the tree, nor the planters, nor the table and chairs were visible. The movies and test probes were rendered using standard textures from Bryce 3-D, and the scene included a ground plane with grassy texture and a sky with clouds. Some of this texture is visible in our figures (e.g., Figure 2), but it was much more apparent in the color versions presented on screen. The movies and test probes were 450 pixels wide $(15.88 \mathrm{~cm}$ with approximately $18^{\circ}$ of visual angle) by 337 pixels tall $(11.89 \mathrm{~cm}$ with approximately $13.5^{\circ}$ of visual angle). Inducing displays depicted a smooth animation of $50^{\circ}$ of a circle (not visual angle) as the camera rotated from the left or right, ending with a view centered on one of the object groups (see Figure 3). The animations were rendered at 24 frames/sec and lasted $1 \mathrm{sec}$. Following a 250 -msec gray blank, a test probe was presented that differed from the final movie view by $0^{\circ}, 1^{\circ}, 2^{\circ}, 3^{\circ}$, or $5^{\circ}$ in both directions (left or right).
A

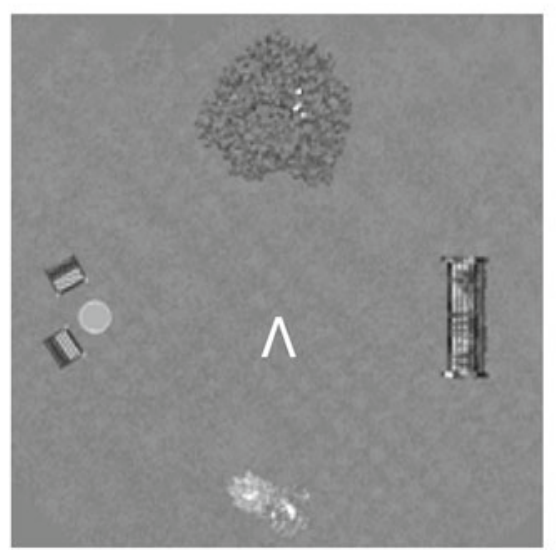

B

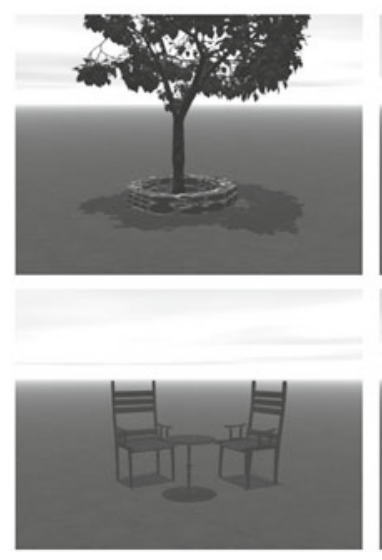

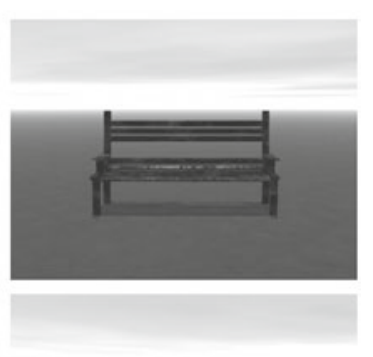

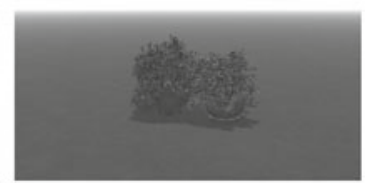

Figure 2. (A) An observer view of the park, with an upside-down white "V" indicating the location of the camera (as shown, rotating across the planters). (B) Centered views of each object, moving clockwise from the top of the map: red-leafed tree, wooden bench, planters (one pot with green plants, the other with pink flowers), and red table and blue chairs. All of the pictures were presented in color, with sky and ground texture, some of which is visible in these grayscale versions. 


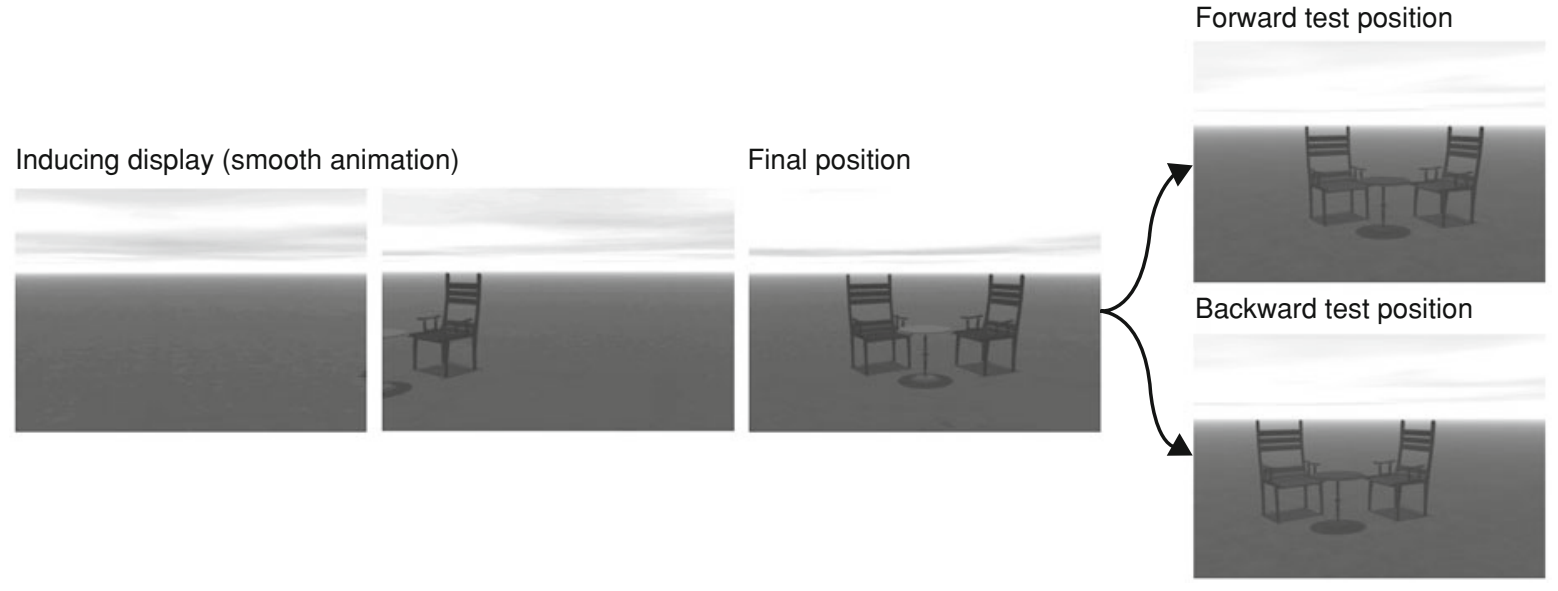

Figure 3. Inducing display of leftward camera rotation, bringing the object group into view from the right. Following the smooth animation, a gray screen appeared and one of nine test probes was presented $\left(+5^{\circ}\right.$ and $-5^{\circ}$ shown here).

Design and Procedure. Each participant viewed 288 experimental trials: 4 object groups (a tree, a table and chairs, two planters, and a bench) $\times 2$ directions (left and right) $\times 9$ test positions $\left(-5^{\circ},-3^{\circ}\right.$, $-2^{\circ},-1^{\circ}, 0^{\circ}, 1^{\circ}, 2^{\circ}, 3^{\circ}$, and $\left.5^{\circ}\right) \times 4$ replications. The participants pressed the return key to watch the movie and then indicated whether or not the presented probe was identical to the final view of the movie by pressing designated keys on the keyboard. To familiarize the participants with the task, 10 representative practice trials with feedback concerning accuracy were presented in a different random order for each participant before the experimental trials. The experimental trials were presented without feedback and in a different random order for each participant. As is standard in RM experiments, the participants were unaware of the unequal probabilities for same and different probes, and instructions emphasized responding on the basis of what the display looked like. As soon as they had finished, the participants were informed of the background and purpose of the experiment.

\section{Results and Discussion}

To estimate the size and direction of the distortion, weighted means were calculated by summing the products of the test positions and the percentage of "same" responses and dividing by the total percentage of "same" responses (see, e.g., Freyd \& Jones, 1994; Hubbard, 1996). This weighted mean is positive when more "same" responses occur for test positions farther along the trajectory, which indicates that the motion has been included in the representation of the event. As revealed by the $95 \%$ confidence intervals, significant positive distortions occurred for all object groups and both directions (see Figure 4). To compare objects and direction, a 4 (object groups) $\times$ 2 (directions) within-subjects ANOVA was computed on
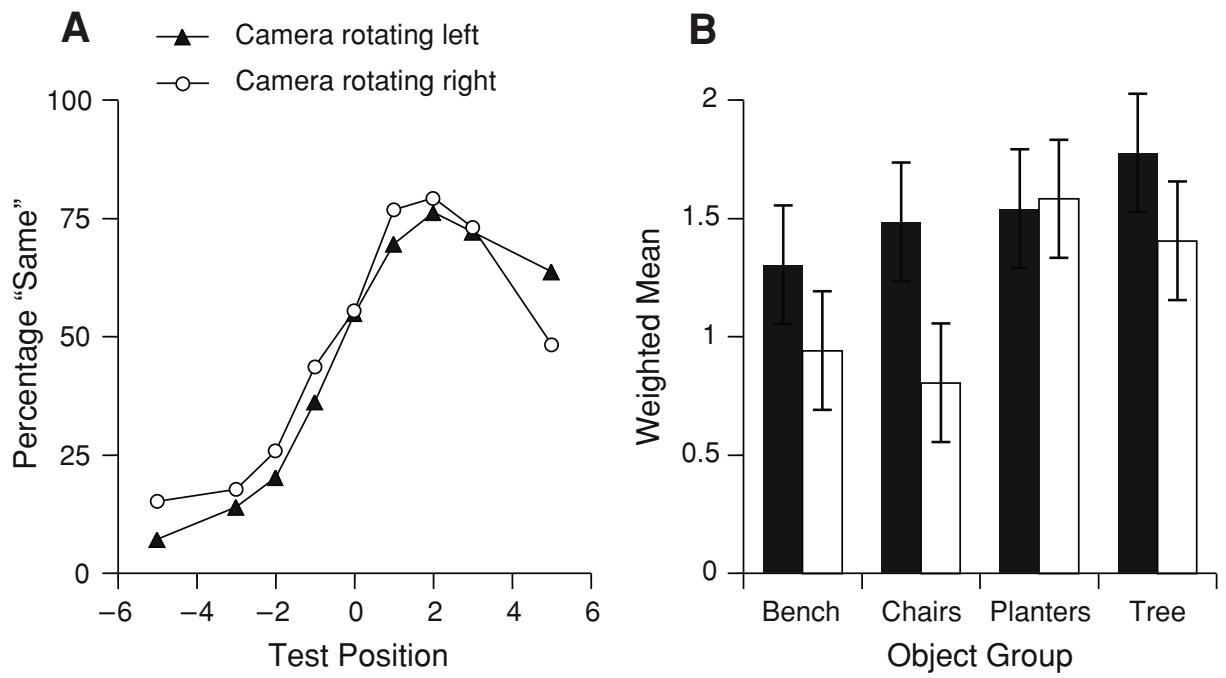

Figure 4. (A) Percentage of "same" responses for camera rotation to the left or right. Test position is measured relative to final view, with positive test positions farther along the depicted rotation. (B) Weighted means for each object group and direction (dark bars for camera rotation to the left, light bars for rotation to the right), with $95 \%$ confidence intervals based on the standard errors of the differences between the means (Loftus \& Masson, 1994) to indicate significance from Experiment 1. 
the weighted means. There was a significant main effect of object group $[F(3,57)=11.93, p<.01]$, and Tukey's HSD analysis revealed that distortions for the bench and table and chairs were smaller than distortions for the tree and the planters $(p<.05)$. Both the tree and the planters have detailed leaf textures, which might have led to more certainty about position and thus subsequent RM, but this was not part of our manipulation. There was also a significant main effect of direction $[F(1,19)=12.02, p<$ $.05]$, with smaller distortions when the camera rotated to the left, and a significant interaction between object group and direction $[F(3,57)=3.06, p<.05]$.

Camera rotations across the scene did lead to RM anticipations of continued movement, with more distortion occurring when the camera was rotating to the left than to the right. When a camera is rotating to the left, the objects are moving across the viewing frame to the right (see Figure 3). Finding larger distortions for leftward camera rotations (which corresponds to objects moving across the frame to the right) replicates Halpern and Kelly (1993), who found more RM for objects moving to the right, although this asymmetry is not always observed (see, e.g., Cooper \& Munger, 1993; Hubbard \& Bharucha, 1988; Munger, Solberg, Horrocks, \& Preston, 1999). We next examined whether participants could recognize an observer map of a scene based on their experience of repeated viewings of the scene as the camera rotated across it and their judgment of the final view.

\section{EXPERIMENT 2}

As Experiment 1 demonstrated, rotating camera movement in a scene leads to RM distortions. These results suggest that participants were developing a dynamic repre- sentation of the movement, but what aspects of the overall scene were included in this representation? The present experiment showed rotations similar to those shown in Experiment 1, and then tested recognition of an observer map of the depicted scene. In addition, half of the participants were explicitly taught the observer map of the scene in advance, to see if knowing the layout would impact RM.

New inducing displays were necessary, since those used in Experiment 1 never showed the relative arrangement of the objects - each animation showed only a single object group. All of the new inducing displays began and ended with partial views of an object group (see Figure 5). Half of the new inducing displays showed the relationship between two object groups; the camera rotation began with a partial view of one object group, and then the rotation brought a new group partially into the viewing frame (see Figure 5A). This partial view of the new object group was the final view of these movies, and the participants were asked to indicate whether a probe presented afterwards was identical to this view. The other half of the new inducing displays began by showing a partial view of a single object group, and then the camera rotation caused the object group to leave the viewing frame (see Figure 5B).

There are several reasons to think that different amounts of RM will occur for camera rotations that bring objects into the picture than for rotations that take objects out of the picture. When the camera rotation is bringing the object into the picture, both boundary extension (BE) and RM support positive distortions, as more of the object group and its background are visible with the positive probes (see Figure 5A). However, when the camera rotation is taking the object out of the picture, BE supports negative distortions, since participants should accept as

\section{A Camera rotation bringing object into viewing frame (smooth animation)}
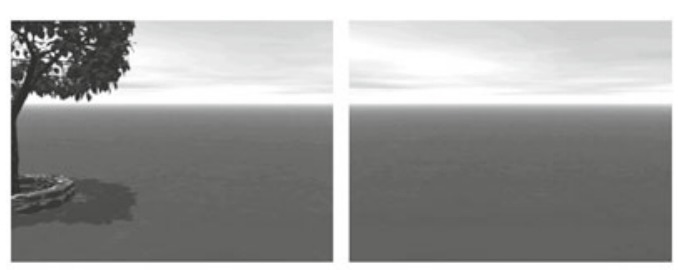

B Camera rotation taking object out of viewing frame (smooth animation)
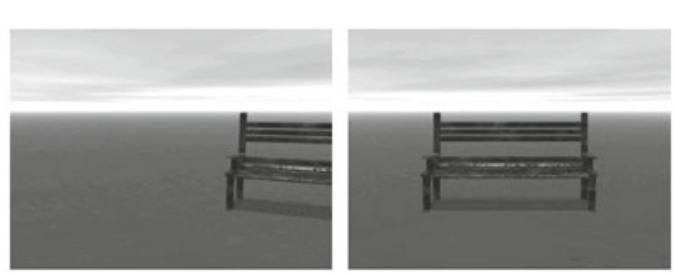

\section{Final position}
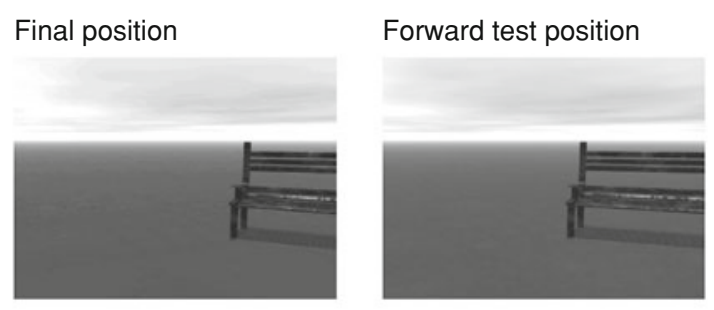

Final position

Backward test position
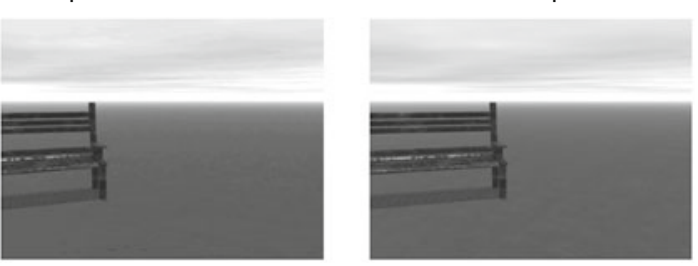

Figure 5. (A) Inducing display of rightward camera rotation bringing the bench into the viewing frame; notice that the animation starts with a partial view of the tree. Following smooth animation, a gray screen appeared and one of nine test probes was presented $\left(+5^{\circ}\right.$ shown here). (B) Inducing display of rightward camera rotation that takes the bench out of the viewing frame, with $-5^{\circ}$ test probe depicted. 
"same" scenes showing more of the object group and background (see Figure 5B). Continuing the motion of the camera so that its view moves beyond the object results in less of the object being visible, so RM is in opposition to BE. Munger et al. (2005) found no relationship between $\mathrm{RM}$ and $\mathrm{BE}$ for depth translations into a scene, but an interaction may result as we explore movement at the edge of the viewing frame.

In addition to a possible interaction with $\mathrm{BE}$, there is evidence from research using simpler objects that the final position of an object that moves into the viewing frame is processed differently from an object that moves out of it. For example, Müsseler and Van der Heijden (2004) found evidence that the visual system is more sensitive to movement that brings objects into view, and thus larger RM distortions may result for objects being brought into the viewing frame compared with those being taken out of it. In addition, when participants are asked to indicate the final position of a small dot or square that disappears near or at the edge of a frame, smaller forward distortions, and sometimes backwards distortions, are observed (Actis-Grosso \& Stucchi, 2003; Hubbard \& Bharucha, 1988; Hubbard \& Motes, 2005). These smaller distortions have been attributed to participants' expectation that the small object would bounce (Hubbard \& Bharucha, 1988) or that they would not see the target outside the boundaries of the frame (Hubbard \& Motes, 2005). However, the inducing displays of the present experiment did nothing to suggest that the camera motion would reverse as the object approached the edge of the viewing frame, and in fact objects were depicted leaving the viewing frame completely (see Figure 5A).

\section{Method}

Participants. Forty-two undergraduates (17 men and 25 women) from the same pool as Experiment 1 participated; none had participated in any experiments with these scene stimuli. Twenty (11 men and 9 women) were in the surprise map condition, and 22 (6 men and 16 women) were in the teach map condition.

Apparatus. The same apparatus as in Experiment 1 were used.

Stimuli. The same park scene as in Experiment 1 was used, but the camera rotation was altered to show object groups being brought into and taken out of the viewing frame during the $50^{\circ}$ sweep of the inducing display (see Figure 5). New test probes were rendered at $-5^{\circ},-3^{\circ},-2^{\circ},-1^{\circ}, 0^{\circ}, 1^{\circ}, 2^{\circ}, 3^{\circ}$, and $5^{\circ}$ surrounding the new final views.

Design and Procedure. The design and procedure were identical to those of Experiment 1 with three exceptions: To reduce the total number of trials, the camera always rotated to the right; a surprise observer map recognition test followed the RM trials; and 22 of the participants were asked to memorize the observer map at the start of the experiment. Before these 22 participants began the RM trials, we tested their memory of the map by showing them a centered picture of one of the object groups from the field view (see Figure 1B), with the question, "Here's the [object group], what is to your right?" on screen. This matched the object group combinations in the inducing displays, resulting from camera rotations to the right, that participants would later see during the RM block. For example, Figure 5 shows the inducing display starting with the tree. As the camera rotates to the right, the tree is taken out of the viewing frame and the bench is brought into the viewing frame. From this sequence, you can see that the bench is to the right of the tree. The participants responded by pressing keys ("b" for bench, "c" for table and chairs, "t" for tree, and " $p$ " for planters), which were designated by a small printed legend taped to the computer screen, and were told whether their answers were correct or incorrect. These four identification trials were repeated until the participants got four correct answers in a row, at which point they received instructions for the RM block.

Each participant viewed $288 \mathrm{RM}$ experimental trials: 2 frames (into and out of the viewing frame) $\times 4$ object groups (a tree, a table and chairs, two planters, and a bench) $\times 9$ test positions $\left(-5^{\circ}\right.$, $-3^{\circ},-2^{\circ},-1^{\circ}, 0,1^{\circ}, 2^{\circ}, 3^{\circ}$, and $\left.5^{\circ}\right) \times 4$ replications. Immediately following the RM trials, the surprise observer map recognition test was automatically presented on screen. The participants were asked to indicate which of two mirror image maps was correct by pressing designated keys (see Figure 1A).

\section{Results and Discussion}

Weighted means were calculated and are presented in Figure 6 along with 95\% confidence intervals (see Experiment 1 for details), and they reveal significant positive distortions for all conditions. A 2 (into and out of the viewing frame) $\times 4$ (object group) mixed ANOVA, with the in-advance map memorization task as a between-subjects variable, was computed on the weighted means. There was a significant main effect of frame $[F(1,40)=10.10, p<$ $.01]$, with larger distortions when camera rotations moved objects into the viewing frame. There was also a significant main effect of object $[F(3,120)=3.62, p<.05]$, but Tukey's HSD analysis revealed no significant differences between any object pair. There was a significant interaction between object group and frame $[F(3,120)=20.31$, $p<.01]$, with the bench showing the opposite pattern from the other three object groups (see Figure 6). Tukey's HSD analysis found significantly larger distortions for the tree and table and chairs than for the bench when camera rotations brought objects into the viewing frame (both $p \mathrm{~s}<.01)$. When camera rotations began to take objects out of the viewing frame, smaller distortions were found for the tree $(p<.01)$ and table and chairs $(p<.05)$ than for the bench. The three object groups (tree, bench, and table and chairs) have similar widths, although the tree is obviously taller (see Figure 2B). The bench was rendered with a grainy wood texture, which is hard to see in the figures but was very clear when presented in color, but it does have fewer internal details than the tree or table and chairs. The lesser detail may have contributed to the different pattern of distortions observed for the bench.

There was no difference in RM between participants who had been asked to memorize the map in advance and those who had $\operatorname{not}(F<1, p>.10)$. However, there was a significant negative correlation between overall RM and successful map recognition at the end of the experiment $(r=-0.34, p<.05)$. Both groups had significant forward distortions, with participants who recognized the map showing significantly smaller RM distortions $[n=$ $32, M=0.50, S D=0.65 ; t(31)=4.68, p<.01]$ than those who did not recognize the map $[n=10, M=1.02$, $S D=0.74 ; t(9)=4.34, p<.01$; between-groups $t(40)=$ $2.26, p<.05]$.

Overall, the correct map was chosen $76 \%$ of the time $( \pm 13,95 \%$ confidence interval, chance at $50 \%)$, which is 

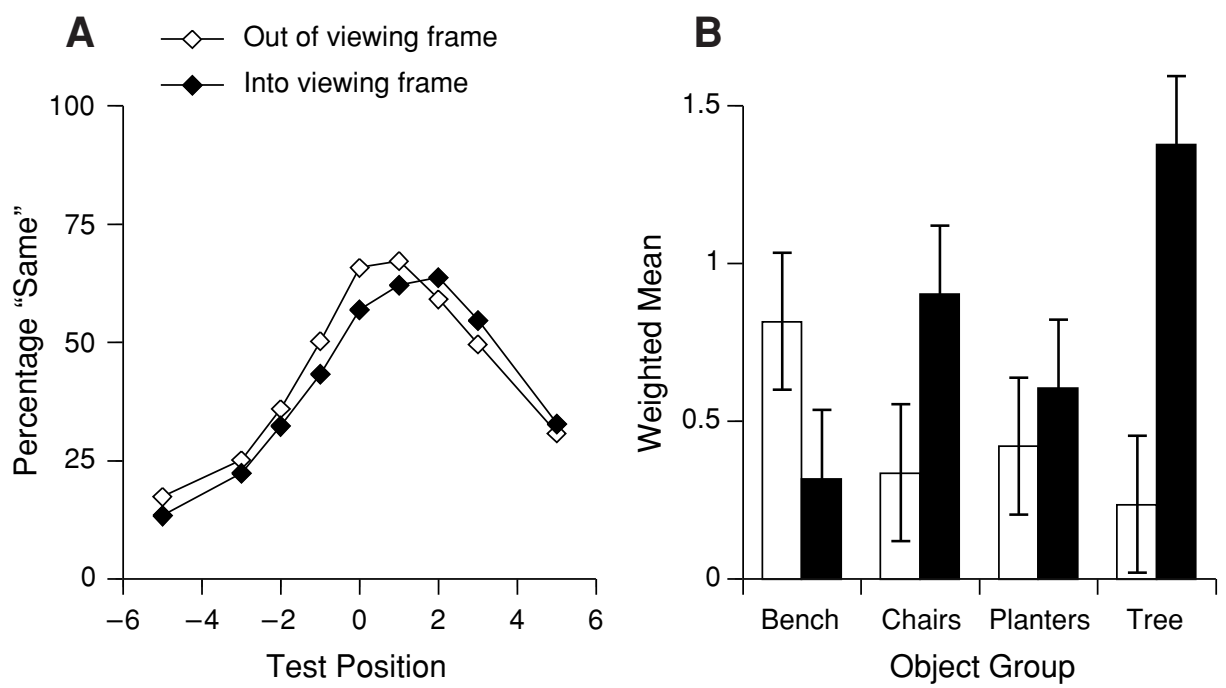

Figure 6. (A) Percentage of "same" responses for camera rotations bringing objects into or out of the viewing frame from Experiment 2 . Test position is measured relative to final view, with positive test positions farther along the camera's rotation. (B) Weighted means for each object group and direction (dark bars for camera motion bringing objects into the viewing frame, light bars for motion taking objects out of the viewing frame), with $95 \%$ confidence intervals based on the standard errors of the differences between the means (Loftus \& Masson, 1994) to indicate significance from Experiment 2.

significantly better than chance. Participants instructed to memorize the map in advance chose the correct map $86 \%$ of the time ( $\pm 15,95 \%$ confidence interval, chance at $50 \%$ ), with only 3 participants ( 2 men, 1 woman) choosing incorrectly. In contrast, participants with no direct experience with the map chose the correct map only $65 \%$ of the time ( $\pm 22,95 \%$ confidence interval, chance at $50 \%)$, which is not better than chance performance. Given the large number of trials, it is quite surprising that participants were unable to extract the correct observer map from their experience with the scene.

RM distortions occurred both when camera rotations brought objects into and when they took objects out of the viewing frame, with larger distortions observed when objects were brought into the viewing frame. This may reflect an interaction with $\mathrm{BE}$ or increased sensitivity to objects entering into our view. Learning the layout of a scene in advance by memorizing an observer map and identifying neighboring object groups using a field view did not alter subsequent RM. However, participants who successfully identified the observer map at the end did have significantly smaller RM distortions than those who did not identify the correct map, regardless of whether they learned the map in advance. We found a negative correlation between RM distortions and map recognition, which might suggest that individuals who were attending to the larger scene (and were thus able to recognize the map) were not attending as much to the immediate position of an object. However, when Hayes and Freyd (2002) examined RM in a divided attention condition, they found that RM increased with divided attention. When considering participants whose only exposure to the scene was through the RM task (the surprise map condition), map recognition was no better than chance, suggesting that the RM task does not lead to an understanding of the larger scene.

\section{EXPERIMENT 3}

Significant RM was observed even when the final view of the object group was partially out of the viewing frame, with larger distortions occurring when the camera rotation brought the object group into the viewing frame (Experiment 2). However, participants without previous exposure to the observer map were unable to correctly identify the scene's map at the end of the experiment. Given the number of RM trials, it was very surprising that participants in the surprise map condition were unable to recognize the map. However, participants may have known more about the spatial arrangement of the scene than this suggests, and hence the present experiment asked about object relations using the same field views as those used in the RM task. Following the RM trials, participants were shown field views of each object group and were asked to identify its left neighbor (see Figure 1B). The inducing displays from the RM trials in the present experiment repeatedly showed the left neighbor for each object group.

\section{Method}

Participants. Twenty-one undergraduates ( 7 men and 14 women) from the same pool as in Experiment 1 participated; none had participated in any experiments with these scene stimuli.

Apparatus. The same apparatus as in Experiment 1 were used.

Stimuli. The same park scene as in Experiments 1 and 2 was used, and the rotation of the camera was manipulated as in Experiment 2 to show object groups being brought into and taken out of the viewing frame. 
Design and Procedure. The design and procedure were identical to those used in the surprise map condition of Experiment 2, but a field view was used for the surprise test of spatial layout (see Figure 1B). The camera always rotated to the left, to reduce the number of trials. Each participant viewed 288 RM experimental trials: 2 frames (into and out of the viewing frame) $\times 4$ object groups (a tree, a table and chairs, two planters, and a bench) $\times 9$ test positions $\left(-5^{\circ},-3^{\circ},-2^{\circ},-1^{\circ}, 0,1^{\circ}, 2^{\circ}, 3^{\circ}\right.$, and $\left.5^{\circ}\right) \times 4$ replications. Immediately following the RM trials, the participants were shown field views of each object group; they then typed their answers to the question, "Here's the [object group], what is to your left?" Each participant completed four identification trials, one for each object group, in a unique random order. The leftward camera movement used for the RM trials presented the object group that was to the left of the final object group.

\section{Results and Discussion}

Weighted means were calculated and are presented in Figure 7 along with 95\% confidence intervals (see Experiment 1 for details), and they reveal significant positive distortions for 5 of 8 frame $\times$ object group combinations. A 2 (into and out of the viewing frame) $\times 4$ (object group) within-subjects ANOVA was computed on the weighted means (see Experiment 1 for details on the weighted mean), and no main effect of frame or object group was found. Collapsed across object groups, significant RM distortions occurred both when camera rotations brought objects into the viewing frame $[M=0.52, S D=0.77$; $t(20)=3.10, p<.01]$ and when camera rotations took objects out of the viewing frame $[M=0.26, S D=0.57$; $t(20)=2.11, p<.05]$, but, as the ANOVA reveals, there was not a difference in RM between frame types. There was a significant interaction between object group and frame $[F(3,60)=11.11, p<.01]$, and Tukey's HSD analysis revealed a larger distortion with the table and chairs compared with the bench when camera rotations brought objects into the viewing frame $(p<.05)$. In addition, when camera rotations took objects out of the viewing frame, both the planters $(p<.01)$ and the table and chairs $(p<.05)$ yielded smaller distortions than the bench. As in Experiment 2, distortions show the opposite pattern for the bench than for the other object groups.

A field view identification score was calculated for each participant by averaging the number of correct answers to the four identification trials at the end of the experiment, and performance was not better than chance $(M=48.8 \%, \pm 16.4,95 \%$ confidence interval, chance at $33 \%$ ), with only 7 of the 21 participants scoring above $50 \%$. This finding is particularly striking because the inducing displays in the RM trials actually showed what was to the left of each object group, yet participants were at chance when asked to indicate an object group's neighbor. In addition, there was no correlation between overall RM and field view identification score.

When camera movement both to the right and to the left was presented in Experiment 1, larger RM was observed with camera movement to the left. To reduce the overall number of trials, single directions were chosen for Experiments 2 and 3, and significant forward distortion was observed both for the rightward camera motion of Experiment $2[M=0.63, S D=0.67 ; t(41)=6.05, p<$ $.01]$ and for the leftward camera motion of the present experiment $[M=0.39, S D=0.49 ; t(20)=3.67, p<.01]$. A between-groups $t$ test revealed no difference between the sizes of these distortions $[t(61)=1.42, p>.10]$. This finding highlights the fact that the rightward asymmetry for object motion found by Halpern and Kelly (1993) is not always observed (see, e.g., Cooper \& Munger, 1993; Hubbard \& Bharucha, 1988; Munger, Solberg, Horrocks, \& Preston, 1999).
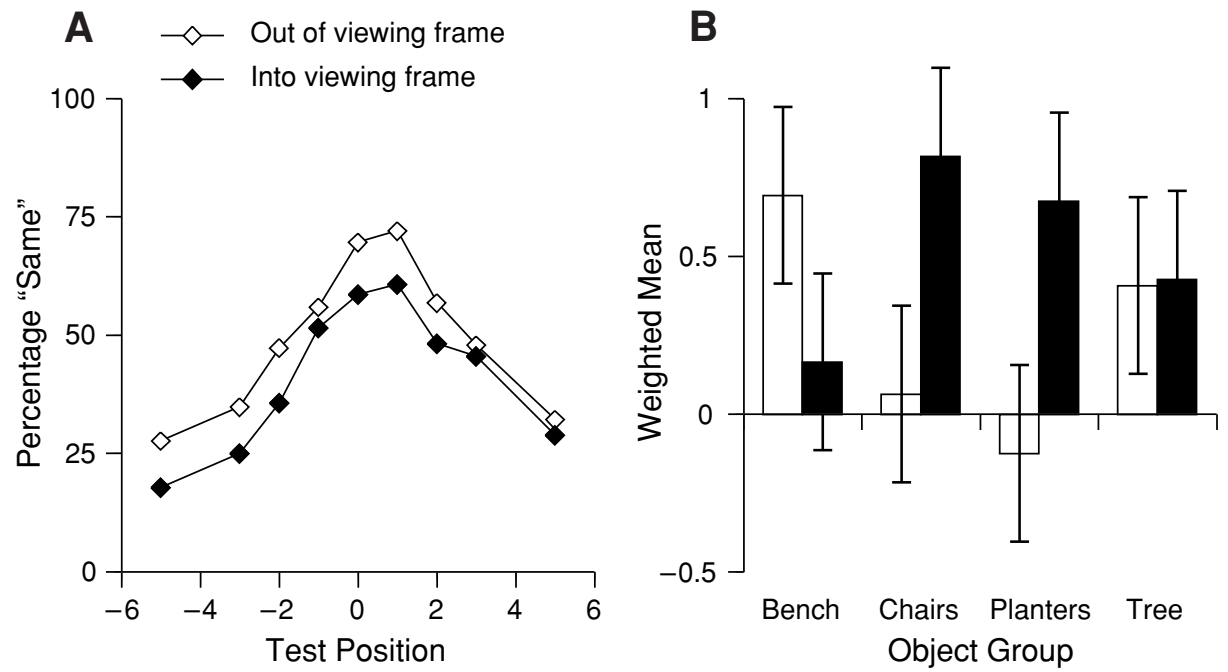

Figure 7. (A) Percentage of "same" responses for camera rotations bringing objects into or out of the viewing frame from Experiment 3. Test position is measured relative to final view, with positive test positions farther along the camera's rotation. (B) Weighted means for each direction (dark bars for camera motion bringing objects into the viewing frame, light bars for motion taking objects out of the viewing frame), with $95 \%$ confidence intervals based on the standard errors of the differences between the means (Loftus \& Masson, 1994) to indicate significance from Experiment 3. 
Significant RM distortions were observed in the present experiment, but participants were at chance when presented with a field view of an object group and asked to indicate what was to the left. It seems as though this would have been a particularly easy task, since the information was presented during the inducing displays when camera rotations brought objects into the viewing frame. Participants could have been asked to compare across RM trials (e.g., "Here's the bench, what's behind you?" "Table and chairs"; see Figure 2A), but given the present results it seems highly unlikely that participants would have been able to respond correctly. Participants with low field view identification scores did not consistently identify object groups to the right, so they were not simply confusing left and right. Repeated viewing of the inducing displays as part of an RM task did not lead to participants' knowing the spatial arrangement of the scene, even when they were prompted with field views identical to views from the RM display.

\section{EXPERIMENT 4}

Camera rotations across a scene lead to RM distortions for view, both when the objects are fully visible (Experiment 1) and when they are partially obscured at the edge of the viewing frame (Experiments 2 and 3). In addition, more RM is observed when camera rotations bring objects into the viewing frame (Experiment 2). However, despite considerable exposure to the scene, participants have little knowledge of the scene's spatial layout. They are unable to recognize the correct observer map, and are not even able to identify an object's neighbor when shown field views identical to those presented during the RM trials.

Wilson and Péruch (2002) found improved performance on map drawing when participants were specifically instructed to attend to spatial layout. Based on this, the present experiment attempted to direct participants' attention to the spatial layout of the scene by embedding a new task within the RM block that explicitly asked them to indicate what was to the left or right of a presented object group. Following this combined block, participants were asked to identify the correct observer map, as in Experiment 2. Given the negative correlation found between successful map recognition and overall RM in Experiment 2, RM may shift as participants attend to the layout to accomplish the new task.

\section{Method}

Participants. Eighteen undergraduates ( 8 men and 10 women) from the same pool as in Experiment 1 participated; none had participated in any experiments with these scene stimuli.

Apparatus. The same apparatus as in Experiment 1 were used.

Stimuli. The inducing displays and test probes from Experiments 2 and 3, which depicted camera rotations that brought object groups into the viewing frame, were used, along with the observer maps.

Design and Procedure. The design and procedure were identical to those used in the surprise map condition of Experiment 2, with the surprise observer map test following the RM trials with two modifications: A new layout task was intermixed with the RM trials, and all the inducing displays showed camera rotations that brought object groups into the viewing frame. Layout trials presented a centered field view of an object group above a question about what was to the left or right, which the participants answered by pressing keys ("b" for bench, "c" for table and chairs, "t" for tree, and "p" for planters) designated by a small printed legend taped to the computer screen. Camera movement to the left and right was included in the RM trials.

Sixteen practice RM trials, with two instances of each camera rotation and object group, preceded the mixed experimental block. Each participant viewed $288 \mathrm{RM}$ experimental trials [2 directions (left and right) $\times 4$ object groups (a tree, a table and chairs, two planters, and a bench) $\times 9$ test positions $\left(-5^{\circ},-3^{\circ},-2^{\circ},-1^{\circ}, 0\right.$, $1^{\circ}, 2^{\circ}, 3^{\circ}$, and $\left.5^{\circ}\right) \times 4$ replications] and 32 layout experimental trials [2 questions ("What is to the left?" "What is to the right?") $\times 4$ object groups (a tree, a table and chairs, two planters, and a bench) $\times$ 4 replications]. Immediately following the combined RM and layout experimental block, the surprise observer map recognition test was automatically presented onscreen. Participants were asked to indicate which of two maps was correct by pressing designated keys (see Figure 1A).

\section{Results and Discussion}

Weighted means were calculated and are presented in Figure 8 along with 95\% confidence intervals (see Experiment 1 for details), and they reveal significant positive distortions for all object groups and directions, except the bench. A 2 (direction) $\times 4$ (object group) within-subjects ANOVA was computed on the weighted means. There was a significant main effect of direction $[F(1,17)=5.27, p<$ $.05]$, with smaller distortions for leftward camera rotations and a significant main effect of object $[F(3,51)=8.67$, $p<.01]$. Tukey's HSD analysis revealed that there was less overall distortion for the bench compared with the tree $(p<.01)$. A similar pattern was observed in Experiment 1 , perhaps as a result of the objects' different textures, although texture was not systematically manipulated.

Significant RM was observed with rightward camera rotations $[M=0.61, S D=0.83 ; t(17)=3.11, p<.01]$ and with leftward camera rotations $[M=0.46, S D=0.77$; $t(17)=2.56, p<.05]$; it is comparable with the matching conditions from Experiment 2 [rightward camera rotations bringing objects into the viewing frame: $M=0.80$, $S D=0.79$; between-groups $t(58)=0.84, p>.10]$ and Experiment 3 [leftward camera rotations bringing objects into the viewing frame: $M=0.52, S D=0.77$; betweengroups $t(37)=0.23, p>.10]$. While the size of the distortions is equivalent to that observed in Experiments 2 and 3 , the direction effect is opposite that observed in Experiment 1, where larger distortions occurred for leftward camera rotations. The direction effect may be more complicated with camera movement across a scene and is not always observed with object motion (see, e.g., Cooper \& Munger, 1993; Hubbard \& Bharucha, 1988; Munger, Solberg, Horrocks, \& Preston, 1999).

Overall accuracy on the layout trials was significantly better than chance $(66 \%, \pm 11,95 \%$ confidence interval, chance at $33 \%$ ). Interestingly, the very first time a layout trial occurred, performance was at chance $(36 \%, \pm 23$, $95 \%$ confidence interval), with improved performance as the experimental block continued. Note that before the first layout trial occurred, the participants had seen each of the possible camera rotations and object groups twice, so they had sufficient information to answer the question 

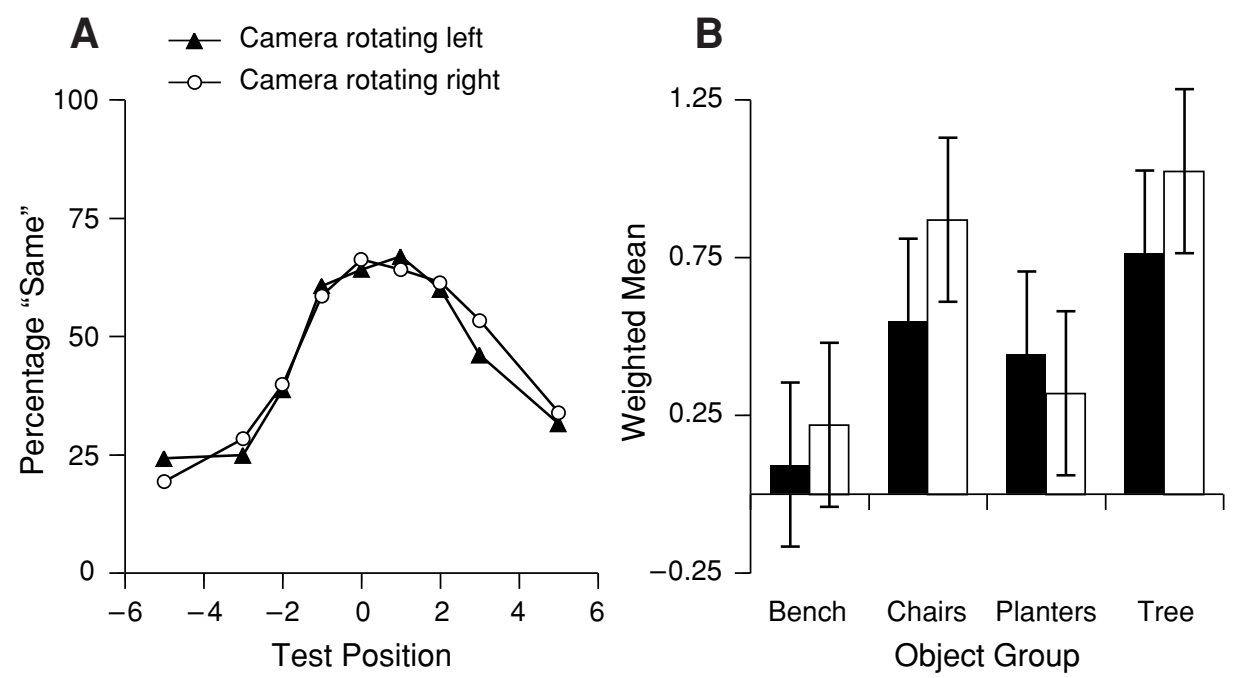

Figure 8. (A) Percentage of "same" responses for camera movements bringing objects into the viewing frame from the left or right from Experiment 4. Test position is measured relative to final view, with positive test positions farther along the depicted rotation. (B) Weighted means for each direction (dark bars for camera rotation to the left, light bars for rotation to the right), with $\mathbf{9 5 \%}$ confidence intervals based on the standard errors of the differences between the means (Loftus \& Masson, 1994) to indicate significance from Experiment 4.

correctly. There was no correlation between RM distortions and layout scores.

Participants identified the correct observer map at better than chance performance $(72 \%, \pm 21,95 \%$ confidence interval, chance at $50 \%$ ), and there was a significant correlation between layout accuracy and map scores $(r=0.52$, $p<.05)$, but no correlation between overall RM distortion and map scores. When participants completed the layout trials, they successfully recognized a map of the scene, which they were not able to do when they completed the RM trials alone (Experiments 2 and 3). Watching a scene from the perspective of a camera rotating across it can allow participants to identify relations between objects, but only when the participants are directly asked to do so. Unlike in Experiment 2, the present experiment found no decrease in RM distortions with successful recognition of the observer map.

\section{GENERAL DISCUSSION}

Making judgments regarding the immediate location of objects in a scene as a camera moves through it is not equivalent to attending to the relative locations of objects in a static scene. With repeated performance of the RM task, participants were unable to learn the layout of the presented scene (Experiments 2 and 3 ). RM was observed in all our experiments, supporting the idea that motion was part of the representation of the event, even in a scene (DeLucia \& Maldia, 2006; Freyd, 1993; Munger et al., 2005; Thornton \& Hayes, 2004). However, repeated exposure to the scene, with attention directed to current details of the movement, did not lead to participants' learning the spatial layout. We tested spatial layout using two perspectives: an observer map and a field view. Successful identification of the observer map required participants to extract an overhead view from the presented field views, which may be a harder task than simply recognizing views. Whereas Janzen et al. (2001) found that participants could readily accomplish a similar task, our participants were at chance for identifying the correct observer map (Experiment 2). More surprising yet, participants were also at chance for identifying objects' neighbors when shown a field view identical to that used in the RM task itself (Experiment 3).

When instructed to learn the map (Experiment 2) or asked direct questions about objects' neighbors during the experimental block (Experiment 4), participants were able to identify the correct observer map at the end of the experiment. In both cases, participants were instructed to attend to the relationships between objects, thus extending the finding of Wilson and Péruch (2002) that instruction affects the quality of learned spatial layout. Apparently, attending to object location within the view of a camera rotating across the scene (the RM task) does not involve attending to object relations within the scene, or even being able to recall them later. Spatial layout was learned only when participants were directed to attend to object relationships within the scene, and even then, only through some repeated exposure. When asked to identify objects' neighbors (Experiment 4), participants were at chance on the very first trial, despite having received complete instructions about the task and having seen each neighboring object group relation as part of the initial RM trials.

Explicitly learning the layout of a scene did not affect RM (Experiment 2). There is clear evidence that the predictability of individual trials increases RM (Kerzel, 2002), although the size of the distortion does not change within an experimental block (Munger \& Owens, 2004). 
We wondered if knowing the layout of the scene would alter RM distortions, particularly for camera rotations that brought objects into the viewing frame, since participants would know exactly which objects would be coming into view once they saw the first object. Participants successfully learned the spatial layout before the RM trials began, correctly identifying each object's neighbor. RM was observed, but it was no different from that observed in the other condition where the spatial layout was not learned (Experiment 2). Knowing the identity of the object about to come into view does not alter RM.

Effects of object group were observed across the experiments, but there was no clear pattern. Positive distortions occurred for each object group, but larger distortions generally occurred for the tree, with smaller distortions for the bench (Experiment 3 is the exception). Larger RM has been observed previously for objects that can move (Vinson \& Reed, 2002), but none of the objects in the park scene could move themselves. In fact, the most immobile of the objects was probably the large tree, which often led to the largest RM. Inspection of the bench, whose distortion pattern was opposite that of the other three object groups (Experiments 2 and 3), suggests that the lack of detail within its interior might have played a role. Whereas the tree has a central trunk, the table and chairs have obvious object boundaries, and the plants in the two pots are different colors, the bench has relatively few internal markings. While this may have contributed to a difference in how participants tracked the bench, it is not clear why it would have led to opposite effects for camera rotations that brought the bench into versus out of the viewing frame.

There is an interesting new asymmetry in the magnitude of RM distortions in the present research: Larger RM distortions were found with camera rotations that brought objects into the viewing frame compared with camera rotations that took objects out of the viewing frame (Experiment 2). One possible reason for this asymmetry is an additive effect between RM and BE. BE occurs when participants claim to remember more of a scene than was actually presented (see, e.g., Intraub, 2002). When considering translations directly into a scene, there appears to be no interaction between RM and BE (DeLucia, 2001; DeLucia \& Maldia, 2006; Munger et al., 2005). However, the BE measure itself shifts after participants have viewed an approach sequence (Munger et al., 2005), suggesting that movement can influence participants' memory for scene boundaries. The present movies depicted camera rotations that caused objects to move into and out of the viewing frame at the edges, unlike in previous studies (DeLucia, 2001; DeLucia \& Maldia, 2006; Munger et al., 2005). At the edges of these movie frames, BE and RM should both be influencing participants' judgments. When the camera rotation brings objects into the viewing frame, both $\mathrm{BE}$ and $\mathrm{RM}$ distortions should lead participants to expect more of the object to be visible. Indeed, camera rotations that brought objects into the viewing frame led to larger positive distortions. However, with camera rotations that take objects out of the viewing frame, BE and RM distor- tions would be in opposition: BE should lead participants to expect more of the object, but RM should lead participants to expect less as the camera rotation continued moving the object out of the viewing frame. The smaller positive RM distortions observed for camera rotations moving objects out of the viewing frame could reflect the opposing expectations resulting from the independent mechanisms of BE and RM.

Smaller, and even backwards, RM for objects heading toward the edge of a frame has been observed previously (Actis-Grosso \& Stucchi, 2003; Hubbard \& Bharucha, 1988; Hubbard \& Motes, 2005), but it is not clear that the proposed reasons for such RM apply to the current work. For example, Hubbard and Bharucha suggested that these smaller distortions reflected participants' expectations that the target object had bounced. Indeed, since exactly that event had been presented in some of their trials, the experimental context supported bouncing events. However, with our park scenes, there was no reason for participants to think that the camera rotations would oscillate. Hubbard and Motes found no systematic distortion for targets moving toward a frame's border, and suggested that participants knew the moving object would not move past this boundary. In contrast, because the inducing displays in which camera rotations eventually brought objects into the viewing frame all began with an object being taken out of the viewing frame, our participants were shown movies that regularly rotated objects out of the viewing frame.

Both Hubbard and Motes (2005) and Actis-Grosso and Stucchi (2003) included another task in their experiments that was not part of our method. In addition to indicating the final position of a moving target (the RM task), their participants were asked to indicate the initial position of a moving target. Distortions for this initial position varied; slower velocity and spatial and directional uncertainty of position led to distortions opposite the motion (the onset repulsion effect; see, e.g., Actis-Grosso \& Stucchi, 2003; Müsseler \& Kerzel, 2004; Thornton, 2002), whereas faster velocity and increased spatial and directional certainty led to forward distortions along the motion (the Fröhlich effect; see, e.g., Hubbard \& Motes, 2005; Müsseler \& Kerzel, 2004). A moving target's initial position can certainly be described as the moment the target comes into view; however, the situation is different when camera rotation brings an object into the viewing frame, the rotation stops, and participants are then asked to indicate the final position of that recently visible object (as in the display and RM tasks in Experiments 2-4). Thus the larger distortions we observed for the final view following camera rotations that brought objects into the viewing frame do not seem like an extension of the Fröhlich effect (forward distortions for the initial view of a moving object).

Judging the position of an object in a scene as a camera rotates the view does not lead participants to develop a sufficient understanding of the spatial layout of the surrounding scene to allow either recognition of the correct observer map or identification of an object's neighbors. Despite hundreds of trials in which participants' only task was to judge the immediate location of an object as a cam- 
era swept past, spatial layout was not learned. When instructed to learn the layout, by either studying an observer map or answering repeated questions concerning objects' near neighbors, participants were able to recognize the observer map. When participants carefully followed the movement of a camera across a scene, the motion was included in their representation of the event, as indicated by the memory distortions in the direction of the depicted motion. The surrounding layout of the scene, however, was not included in the representation of the event. An intriguing new RM asymmetry for objects entering and leaving the viewing frame was observed. We are capable of anticipating continued motion in a scene, but the nature of the motion affects our anticipations.

\section{REFERENCES}

Actis-Grosso, R., \& Stucchi, N. (2003). Shifting the start: Backward mislocation of the initial position of a motion. Journal of Experimental Psychology: Human Perception \& Performance, 29, 675-691.

Christou, C. G., \& BülthofF, H. H. (1999). View dependence in scene recognition after active learning. Memory \& Cognition, 27, 996-1007.

COOPER, L. A., \& Munger, M. P. (1993). Extrapolating and remembering positions along cognitive trajectories: Uses and limitations of analogies to physical motion. In N. Eilan, R. McCarthy, \& B. Brewer (Eds.), Spatial representation: Problems in philosophy and psychology (pp. 112-131). Cambridge, MA: Blackwell.

DeLucIA, P. R. (2001, November). Visual memory for moving scenes: Boundary extension or representational momentum? Poster presented at the 42nd Annual Meeting of the Psychonomic Society, Orlando, FL.

Delucia, P. R., \& Maldia, M. M. (2006). Visual memory for moving scenes. Quarterly Journal of Experimental Psychology, 59, 340-360.

FREYD, J. J. (1993). Five hunches about perceptual processes and dynamic representations. In D. E. Meyer \& S. Kornblum (Eds.), Attention and performance XIV: Synergies in experimental psychology, artificial intelligence, and cognitive neuroscience (pp. 99-119). Cambridge, MA: MIT Press.

FreYd, J. J., \& FINKE, R. A. (1984). Representational momentum. Journal of Experimental Psychology: Learning, Memory, \& Cognition, 10, 126-132.

FREYD, J. J., \& Jones, K. T. (1994). Representational momentum for a spiral path. Journal of Experimental Psychology: Learning, Memory, \& Cognition, 20, 968-976.

Gaunet, F., Vidal, M., Kemeny, A., \& Berthoz, A. (2001). Active, passive and snapshot exploration in a virtual environment: Influence on scene memory, reorientation and path memory. Cognitive Brain Research, 11, 409-420.

Gottesman, C. V., \& Intraub, H. (2004). Constraints on spatial extrapolation in the mental representation of scenes: View-boundaries vs. object-boundaries. Visual Cognition, 10, 875-893.

Halpern, A. R., \& KelL Y, M. H. (1993). Memory biases in left versus right implied motion. Journal of Experimental Psychology: Learning, Memory, \& Cognition, 19, 471-484.

HAYES, A. E., \& FREYD, J. J. (2002). Representational momentum when attention is divided. Visual Cognition, 9, 8-27.

HuBbARD, T. L. (1995). Environmental invariants in the representation of motion: Implied dynamics and representational momentum, gravity, friction, and centripetal force. Psychonomic Bulletin \& Review, 2, 322-338.
Hubbard, T. L. (1996). Displacement in depth: Representational momentum and boundary extension. Psychological Research, 59, 33-47.

Hubbard, T. L. (2005). Representational momentum and related displacements in spatial memory: A review of the findings. Psychonomic Bulletin \& Review, 12, 822-851.

HUBbard, T. L., \& BHARUCHA, J. J. (1988). Judged displacement in apparent vertical and horizontal motion. Perception \& Psychophysics, 44, 211-221.

HubBard, T. L., \& Motes, M. A. (2005). An effect of context on whether memory for initial position exhibits a Fröhlich effect or an onset repulsion effect. Quarterly Journal of Experimental Psychology, 58A, 961-979.

Hubbard, T. L., \& Ruppel, S. E. (1999). Representational momentum and the landmark attraction effect. Canadian Journal of Experimental Psychology, 53, 242-255.

INTRAUB, H. (2002). Anticipatory spatial representation of natural scenes: Momentum without movement? Visual Cognition, 9, 93-119.

INTRAUB, H. (2004). Anticipatory spatial representation of 3D regions explored by sighted observers and a deaf-and-blind-observer. Cognition, 94, 19-37.

JanZEN, G., SchADE, M., KATZ, S., \& Herrmann, T. (2001). Strategies for detour finding in a virtual maze: The role of the visual perspective. Journal of Environmental Psychology, 21, 149-163.

JoRDAN, J. S., \& KNOBLICH, G. (2004). Spatial perception and control. Psychonomic Bulletin \& Review, 11, 54-59.

KERZEL, D. (2002). A matter of design: No representational momentum without predictability. Visual Cognition, 9, 66-80.

Loftus, G. R., \& Masson, M. E. J. (1994). Using confidence intervals in within-subject designs. Psychonomic Bulletin \& Review, 1, 476490.

MûnGer, M. P., \& Owens, T. R. (2004). Representational momentum and the flash-lag effect. Visual Cognition, 11, 81-103.

Munger, M. P., Owens, T. R., \& Conway, J. E. (2005). Are boundary extension and representational momentum related? Visual Cognition, 12, 1041-1056.

Munger, M. P., Solberg, J. L., \& Horrocks, K. K. (1999). The relationship between mental rotation and representational momentum. Journal of Experimental Psychology: Learning, Memory, \& Cognition, 25, 1557-1568.

Munger, M. P., Solberg, J. L., Horrocks, K. K., \& Preston, A. S. (1999). Representational momentum in depth: Effects of axis and shading. Journal of Experimental Psychology: Learning, Memory, \& Cognition, 25, 157-171.

MÜsSELER, J., \& KERZEL, D. (2004). The trial context determines adjusted localization of stimuli: Reconciling the Fröhlich and onset repulsion effects. Vision Research, 44, 2201-2206.

Müsseler, J., \& VAN DER HEIJDEN, A. H. C. (2004). Two spatial maps for perceived visual space: Evidence from relative mislocalizations. Visual Cognition, 11, 235-254.

THORNTON, I. M. (2002). The onset repulsion effect. Spatial Vision, 15, 219-243

THORNTON, I. M., \& HAYES, A. E. (2004). Anticipating action in complex scenes. Visual Cognition, 11, 341-370.

ThORNTON, I. M., \& HubBARD, T. L. (2002). Representational momentum: New findings, new directions. Visual Cognition, 9, 1-7.

VInson, N. G., \& ReED, C. L. (2002). Sources of object-specific effects in representational momentum. Visual Cognition, 9, 41-65.

Wilson, P. N., \& PÉRUCH, P. (2002). The influence of interactivity and attention on spatial learning in a desk-top virtual environment. $\mathrm{Ca}$ hiers de Psychologie Cognitive/Current Psychology of Cognition, 21, 601-633.

(Manuscript received August 6, 2004; revision accepted for publication July 25,2005 .) 